\title{
Adenosine-induced atrial fibrillation during fractional flow reserve measurement
}

\author{
Eugene Park, Adam Price, Mladen I. Vidovich \\ Department of Medicine, Division of Cardiology, University of Illinois at Chicago, Chicago, Illinois, USA
}

\begin{abstract}
We present the case of a 44 year-old man referred for coronary angiography with a history of coronary artery disease. Following administration of intra-coronary adenosine for fractional flow reserve (FFR) assessment, the patient developed atrial fibrillation $(A F)$. While well described during non-invasive stress testing, this is the first description in the literature of $A F$ following adenosine administration for FFR. The growing usage of FFR during diagnostic coronary angiography makes this an important side effect to consider. Our subsequent management and follow-up is presented. (Cardiol J 2012; 19, 6: 650-651)
\end{abstract}

Key words: atrial fibrillation, adenosine, fractional flow reserve

\section{Introduction}

A 44 year-old-man was referred for cardiac catheterization because of symptoms of worsening exertional chest pain. The patient had required multiple percutaneous interventions in the past. Aside from his coronary history, the patient had been treated for diabetes mellitus, hypertension and hyperlipidemia. His medications included aspirin $81 \mathrm{mg}$ daily, clopidogrel $75 \mathrm{mg}$ daily, gemfibrozil $600 \mathrm{mg}$ twice daily, simvastatin $80 \mathrm{mg}$ daily, amlodipine $5 \mathrm{mg}$ daily, carvedilol $25 \mathrm{mg}$ twice daily, lisinopril $40 \mathrm{mg}$ daily, isosorbide mononitrate $30 \mathrm{mg}$ daily, and insulin. He denied any prior history of arrhythmia, dizziness, palpitations or syncope. His pre-catheterization electrocardiogram revealed normal sinus rhythm (SR) with a normal QT interval, and echocardiography demonstrated normal ejection fraction with apical septal hypokinesis.

Prior to coronary angiography, blood pressure (BP) was $126 / 61 \mathrm{~mm} \mathrm{Hg}$ and heart rate was $81 \mathrm{bpm}$. Telemetry demonstrated normal SR. Angiography revealed progression of his underlying coronary disease, with multiple non-obstructive lesions. The most severe lesion was seen in the proximal pos- terior descending coronary artery (PDA), visually estimated at $60-70 \%$ stenosis. Given his clinical presentation of exertional angina, and the intermediate nature of the stenosis, the decision was made to pursue additional functional testing with fractional flow reserve (FFR).

FFR measurement was performed with a Volcano 0.014" pressure wire (PrimeWire ${ }^{\mathrm{TM}}$, Volcano Corporation, Rancho Cordova, CA, USA). During his FFR assessment, $70 \mu \mathrm{g}$ of intra-coronary adenosine was administered. The patient initially developed transient third degree atrioventricular (AV) block during adenosine administration, and following resolution of the AV block, spontaneously developed atrial fibrillation (AF) with rapid ventricular response following a series of premature atrial contractions (PACs) (Fig. 1) The FFR measurement was 0.73 , a clinically significant value, and the patient subsequently received a drug eluting stent to his PDA. The patient remained asymptomatic and hemodynamically stable with a BP of $113 / 69$ and a pulse of $112 \mathrm{bpm}$. He did, however, remain in $\mathrm{AF}$, and the decision was made to admit the patient to the telemetry floor for further monitoring and management of his arrhythmia. Intravenous amiodarone,

Address for correspondence: Mladen I. Vidovich, MD, Department of Medicine, Division of Cardiology, University of Illinois at Chicago, 840 South Wood Street, MC 715, Suite 935, Chicago, IL 60612-7323, USA, tel: 312413 4951, fax: 312 413 2948,

e-mail:miv@uic.edu

Received: 02.11.2011 Accepted: 07.11.2011 


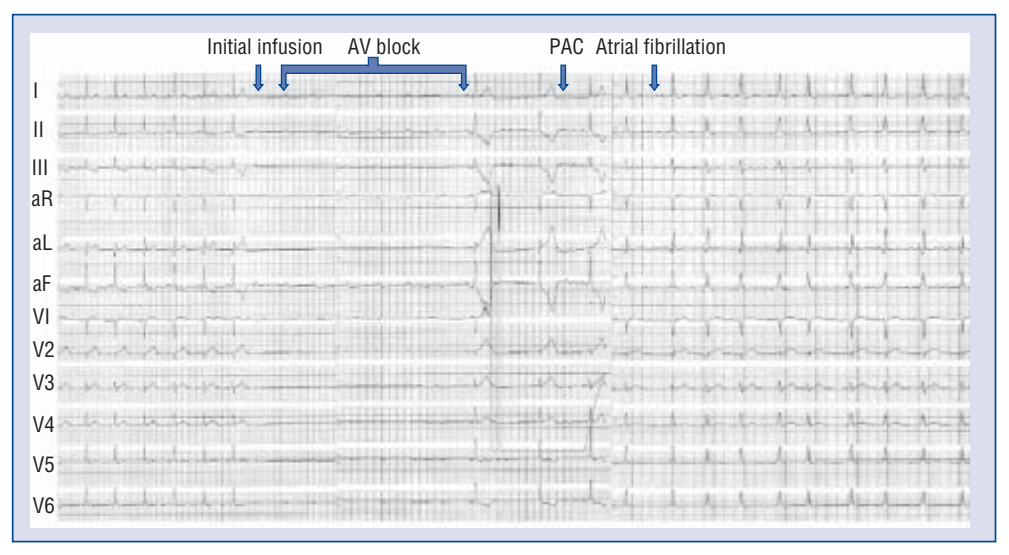

Figure 1. Continuous telemetry recording performed during coronary angiography and adenosine administration. Telemetry reveals initial normal sinus rhythm, followed by transient third degree atrioventricular (AV) block. Following resolution of $\mathrm{AV}$ block, a premature atrial contraction (PAC) occurs, which triggers the development of atrial fibrillation.

$150 \mathrm{mg}$ bolus was administered, followed by a continuous infusion, with a plan to perform electrical cardioversion the following morning should he remain in AF. Aspirin, $325 \mathrm{mg}$ orally, clopidogrel $75 \mathrm{mg}$ orally, as well as his home dose of carvedilol $25 \mathrm{mg}$ twice daily were administered. The patient successfully converted to SR by the following morning, and was discharged home on full dose aspirin, clopidogrel, and the remainder of his home medications.

\section{Discussion}

To the best of our knowledge, this is the first reported case of AF associated with adenosine infusion during FFR assessment. There is already a vast body of literature supporting the usefulness of FFR in the assessment of intermediate lesions, as well as the improved clinical outcomes associated with its usage [1]. The expectation is that FFR and adenosine administration during coronary angiography will become more widespread as a result of this evidence. To date, there is already a well-established link between adenosine and its ability to stimulate cardiac arrhythmias [2]. Adenosine infusion used for the diagnosis of coronary disease has confirmed this association, with AF induction following adenosine myocardial perfusion stress testing having been described previously [3]. The mechanism of this association has been attributed to adenosine's ability both to decrease atrial refractory periods as well as to shorten the action potential duration, making the atrial myocardium more susceptible to arrhythmia induction [2, 4]. In addition, evidence also links adenosine administration to the induction of PACs, which has been clearly linked to the induction of $\mathrm{AF}$ [2].

Currently, there is no reported incidence of this complication related to FFR assessment; however, the morbidity associated with AF makes this an important side effect to consider during functional assessment of intermediate coronary lesions. It is uncertain whether this complication may be more frequently encountered with intracoronary or intravenous adenosine administration. Additionally, the impact of the dose of intracoronary adenosine, administration to the left or right coronary system, coronary dominance or amount of ischemic myocardium on the induction of AF is unknown.

\section{Conclusions}

This case represents a well-described side effect related to adenosine infusion, albeit in a unique setting of FFR assessment. With the growing frequency of FFR assessment during coronary angiography, although rare, the initiation of AF with intracoronary adenosine may be increasingly encountered.

\section{Conflict of interest: none declared}

\section{References}

1. Tonino PA, De Bruyne B, Pijls NH et al. Fractional flow reserve versus angiography for guiding percutaneous coronary intervention. N Engl J Med, 2009; 360: 213-224.

2. Belhassen B, Glick A, Laniado S. Comparative clinical and electrophysiologic effects of adenosine triphosphate and verapamil on paroxysmal reciprocating junctional tachycardia. Circulation, 1988; 77: 795-805.

3. Kanei Y, Hanon S, Van-Tosh A, Schweitzer P. Adenosine-induced atrial fibrillation during pharmacologic stress testing: Report of eight cases and review of the literature. Int J Cardiol, 2008; 129: e15-e17.

4. Kabell G, Buchanan LV, Gibson JK, Belardinelli L. Effects of adenosine on atrial refractoriness and arrhythmias. Cardiovasc Res, 1994; 28: 1385-1389. 